\title{
Pre-Stenting for Retrograde Intrarenal Surgery- Need and Duration: a Prospective Randomized Clinical Study
}

\author{
Prakash Chhettri ${ }^{1}$, Anil Shrestha ${ }^{1}$, Robin Bahadur Basnet ${ }^{1}$, Parash Mani \\ Shrestha ${ }^{1}$
}

${ }^{1}$ Department of Urology, National Academy of Medical Sciences, Bir Hospital, Kathmandu, Nepal

\section{ABSTRACT}

Introduction: To evaluate if retrograde intrarenal surgery with ureteral access sheath requires prestenting. In case pre-stenting becomes an option, how long does it need to be stented.

\begin{abstract}
Materials and Methods: After obtaining approval from Institutional Review Board and informed consent, a prospective randomized controlled study was conducted in the Department of Urology, Bir Hospital for 18 months. All the patients enrolled for retrograde intrarenal surgery were grouped into 3 groups: Group 1 - without pre-stenting, Group 2 - one week of pre-stenting, and Group 3 - two weeks of pre-stenting. Success was defined as an ability to accommodate a 10/12 F ureteral access sheath during retrograde intrarenal surgery. Ureteral access sheath induced ureteric wall injury was also taken into consideration.
\end{abstract}

Results: Among 179 cases, 152 cases were included in the study. In 36 patients out of 53 (67.92\%) in group 1, 10/12 F ureteral access sheath was negotiable without pre-stenting, marking the frequency of distensible ureters. In 44 patients out of 47 (93.66\%) from group 2 and all 52 patients $(100 \%)$ from group 3, ureteral access sheath placement was successful after one and two weeks of pre-stenting respectively. Ureteric wall injury of grade1 and 2, was found in 9 patients (5.9\%).

\section{Correspondence:}

Dr. Prakash Chhettri, MCh (Urology)

Department of Urology,

National Academy of Medical Sciences, Bir Hospital, Kathmandu, Nepal

ORCID ID: 0000-0001-9444-2851

Email: pchhettri@gmail.com

Submitted: $24^{\text {th }}$ November 2020 Accepted: $20^{\text {th }}$ December 2020

Source of Support: None

Conflict of Interest: None

Citation: Chhettri P, Shrestha P, Basnet RB, Shrestha PM. Pre-stenting for retrograde intrarenal surgery -need and duration: a prospective randomized clinical study. NMJ 2020;3(2):361-5. DOI $10.3126 / \mathrm{nmj}$. v3i2.33050

Conclusions: The majority of ureters (67.92\%) are distensible, not requiring pre-stenting before retrograde intrarenal surgery. One and two weeks of pre-stenting carries a success rate of $93.66 \%$ and $100 \%$ respectively.

Keywords: Pre-stenting; Retrograde intrarenal surgery; Ureteral access sheath

\section{INTRODUCTION}

Retrograde intrarenal surgery (RIRS), currently regarded as one of the first-line treatments for renal stone $<2 \mathrm{~cm} .{ }^{1}$ With the advancement in endoscopic techniques in terms of miniaturization and increased flexibility, RIRS has become widely accepted and has further been facilitated by the use of ureteral access sheath (UAS). ${ }^{2,3}$ Insertion of UAS is not free of complication and about $46.5 \%$ of direct ureteral injury has occurred during its negotiation in the ureter. ${ }^{4}$ To overcome this avoidable complication, the ureter can be dilated by active or passive means, prior to the placement of UAS. ${ }^{5}$

The active form of dilatation by various dilators produces linear shearing force with incisions in the ureteric mucosa, resulting in extravasation of irrigation fluid and urine is believed to cause fibrosis. ${ }^{6}$ Pre-stenting has the advantage of passively dilating the ureter allowing for easier access to the upper urinary tract and 
reduces the ureteral trauma. ${ }^{7}$ In addition, a postoperative ureteral stent can be eliminated after RIRS in pre-stented patients. ${ }^{8}$ However, pre-stenting entails a two-stage procedure and bears its own cost. ${ }^{9}$ In addition, stent-related symptoms appear to be the most distressing condition in $90 \%$ of cases. ${ }^{10}$ Even one week of stenting has resulted in reduced quality of life in $80 \%$ of patients. ${ }^{11}$

Since there has not been any consensus about the need and duration of pre-stenting before RIRS, we aim to evaluate prospectively if pre-stenting seems to be mandatory.

\section{MATERIALS AND METHODS}

This prospective randomized control study was conducted in the Department of Urology, National Academy of Medical Sciences, Bir Hospital between period of May 2017 to October 2018. Ethical clearance was obtained from the Institutional Review Board. Patients were randomized into 3 groups according to the computer-generated random numbers. Group 1 underwent RIRS without pre-stenting whereas group 2 and 3 have been prestented for 1 and 2 weeks respectively. Success was defined as an ability to accommodate UAS of 10/12 F during RIRS. When the introduction of UAS failed, then the patient was pre-stented for one more week.
All the patients with nephrolithiasis undergoing RIRS were included in the study. The sample size calculated was 180 (60 in each group; with $98 \%$ power and $95 \%$ confidence level). The exclusion criteria were patients not consenting for the study, age below 14 years, conditions requiring preterm removal of Double J (DJ) stents, previously stented patients (before enrollment), those who have a history of surgery in the ureter, and those who are under alfa-blockers.

Pre-operatively patients were assessed in Urology OPD of Bir Hospital. Demographic parameters of the patients, history, and physical examination were recorded. The patients underwent routine preoperative investigations and a pre-anesthetic checkup. CT Intravenous Urography, with stone density measured in Hounsfield Unit, was done for all the patients. All patients should have sterile urine before the procedure.

Patients were counseled about the study and written informed consent was taken. They were then randomized into 3 groups and scheduled accordingly. Surgery was performed by a single experienced surgeon. The study was conducted in accordance with the Consolidated Standards of Reporting Trials (CONSORT) 2010 statement. $^{12}$ (fig.1)

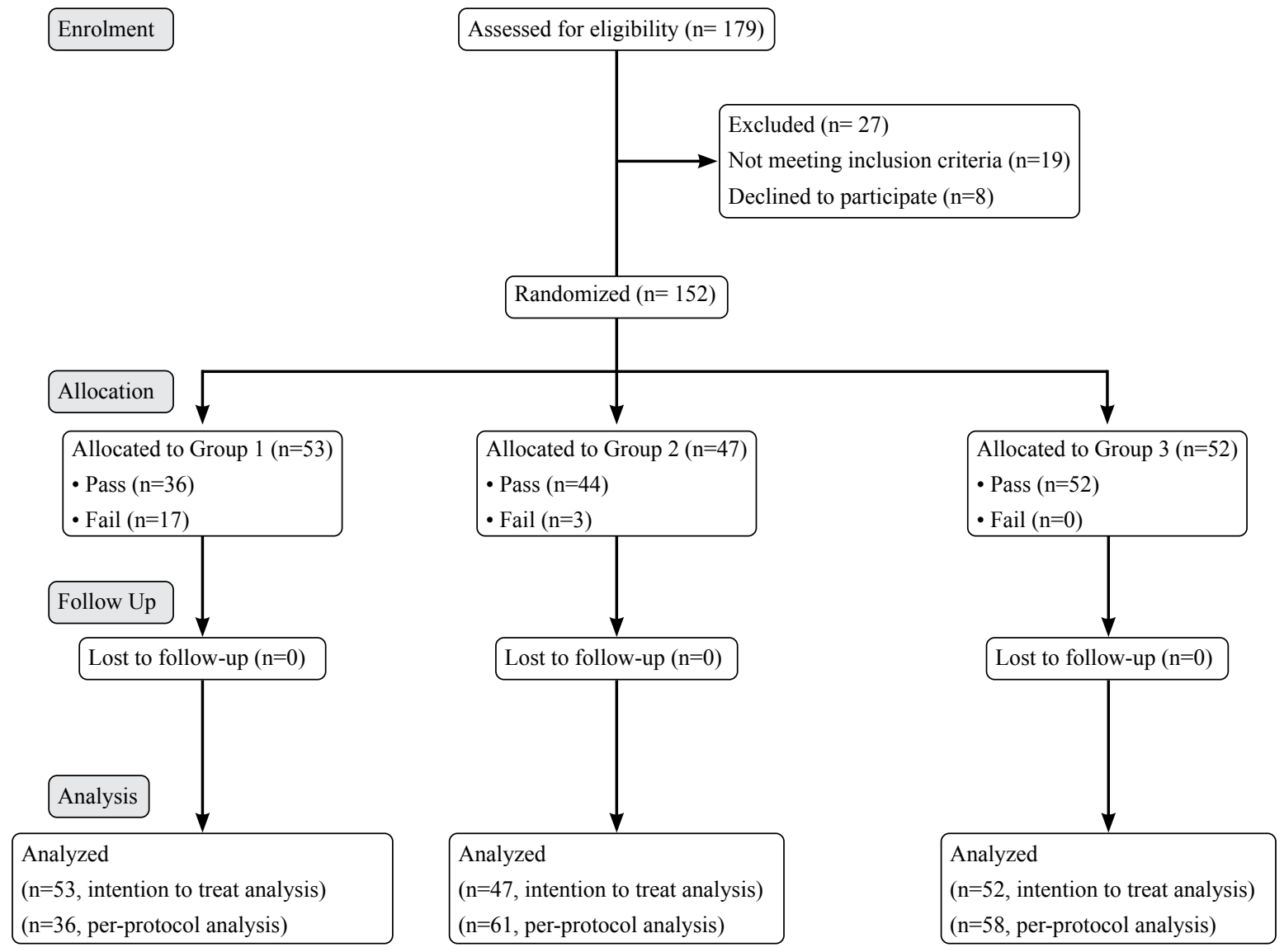

Figure 1: Study cohort diagram 
Pre-stenting was performed in local anesthesia with a prophylactic antibiotic (Inj Ceftriaxone $1 \mathrm{gm}$ ) in a lithotomy position. Cystoscopy was done and the desired ureter was intubated with a 0.035 " glide wire. DJ stent $(6 \mathrm{~F} / 26 \mathrm{~cm})$ was inserted over the guidewire under fluoroscopic guidance, such that the upper coil of the DJ stent lied inside the pelvicalyceal system and the lower coil in the bladder. The patient was then called up for RIRS according to the group they belonged. The patients in group 1 and 2 were counseled that if UAS (10/12 F) cannot be inserted during RIRS on the day, they will be postponed for one more week with DJ stent.

On the day of RIRS, after receiving a prophylactic antibiotic (Inj. Ceftriaxone $1 \mathrm{gm} \mathrm{IV),} \mathrm{a} \mathrm{cystoscope} \mathrm{was} \mathrm{inserted} \mathrm{under} \mathrm{sedation.}$ The tip of the DJ stent (in Group 2 and 3) in the bladder was delivered just beyond the external meatus, the glide wire 0.035 " (Radifocus, Terumo) was introduced into it and the DJ stent removed. Then (and if DJ was not placed), the UAS (Uropass, Olympus) $10 / 12 \mathrm{~F}$ was inserted over the guidewire. If the UAS could be negotiated, then RIRS was continued under general anesthesia. If not, then the RIRS would be postponed for next week, with the re-insertion of the DJ stent. Failure of UAS negotiation could be explained by tenting of the guidewire at the vesicoureteric junction (VUJ) on fluoroscopy.

RIRS in our department was done by flexible ureteroscope Flex$\mathrm{X}^{2 \mathrm{~S}}$ and Flex Xc (Karl Storz) with UAS (Uropass, Olympus) 10/12 F, using 200 micron Ho: YAG laser (Lumenis). We prefer dusting $(0.3 \mathrm{~J}, 60 \mathrm{~Hz}$, long pulse width) and pop-dusting $(0.5 \mathrm{~J}$, $50 \mathrm{~Hz}$, short pulse width) mode of lithotripsy. In the end, DJ stent was kept. And the follow up was done after 2-4 weeks with X-ray KUB and USG of KUB. Any complications during the insertion of UAS were recorded as proposed by Traxer et al. ${ }^{4}$

\section{Statistical Analysis}

All the variables were plotted in Microsoft Excel 2016. Data analysis was done using the statistical package for social sciences, SPSS. All the baseline characteristics and variables were compared using cross-tabulation. The statistical analysis has been presented in tables, charts, and graphs. Patient demographic and clinical characteristics among the groups were compared and a p-value was obtained for each according to Z-test. A p-value $<0.05$ was considered statistically significant.

\section{RESULTS}

Among 179 cases enrolled in the study, twenty-seven cases were excluded due to various reasons. The remaining 152 cases were randomized into three groups, as shown in table 1 .
Table 1: Demographic characteristics

\begin{tabular}{lllll}
\hline & $\begin{array}{l}\text { Group 1 } \\
(\mathbf{n = 5 3 )}\end{array}$ & $\begin{array}{l}\text { Group 2 } \\
(\mathbf{n = 4 7 )}\end{array}$ & $\begin{array}{l}\text { Group 3 } \\
(\mathbf{n = 5 2 )}\end{array}$ & p-value \\
\hline $\begin{array}{l}\text { Age - Mean } \pm \\
\text { SD (years) }\end{array}$ & $37.26 \pm 10.68$ & $39 \pm 15$ & $39 \pm 14$ & 0.748 \\
\hline Male & $32 / 53$ & $29 / 47$ & $33 / 52$ & 0.948 \\
& $(60.37 \%$ & $(61.70 \%)$ & $(63.46 \%)$ & \\
\hline Female & $21 / 53$ & $18 / 47$ & $19 / 52$ & \\
& $(39.62 \%)$ & $(38.29 \%)$ & $(17.30 \%)$ & \\
\hline Right Kidney & $25 / 53$ & $29 / 47$ & $23 / 52$ & 0.182 \\
& $(47.16 \%)$ & $(61.70 \%)$ & $(44.23 \%)$ & \\
\hline Left Kidney & $28 / 53$ & $18 / 47$ & $29 / 52$ & \\
& $(52.83 \%)$ & $(38.29 \%)$ & $(55.76 \%)$ & \\
\hline
\end{tabular}

As depicted in figure 2, Thirty-six out of 53 patients (67.92\%) in group 1 were able to negotiate ureteral access sheath of $10 / 12$ $\mathrm{F}$ in the initial attempt, marking the frequency of distensible ureters. In seventeen patients from group 1 and three patients from group 2, negotiation of UAS was failed and were posted for the subsequent week after stenting. None of the cases in group 3 had been failed to accommodate UAS. In group 1, the mean age of patients who have passed and failed in accommodating UAS was 38.74 years and 32.30 years respectively. Among the failed patients, 12 out of 17 patients in group 1 and all 3 patients in group 2 were males, which is clinically significant $(\mathrm{p}<0.01)$.

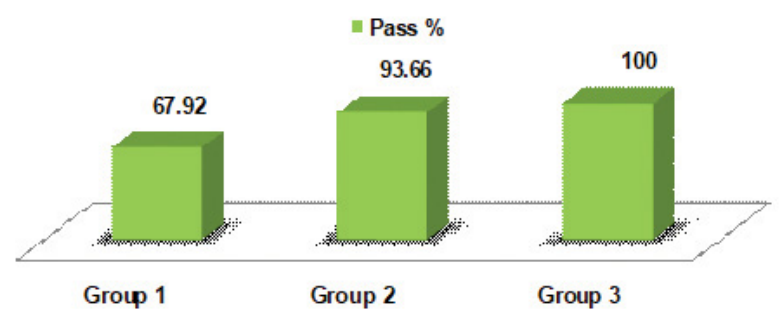

Figure 2. Groupwise analysis on the initial attempt.

Out of 17 patients from group 1, in whom initial passage of UAS was unsuccessful, 14 patients successfully underwent the procedure with 10/12 F UAS after one week of stenting whereas 3 patients required 2 weeks of stenting. Similarly, 3 patients out of 47 patients in group 2 required an additional one week of presenting for the successful passage of 10/12 F UAS. There were no significant differences among the groups according to Intention-to-treat analysis and Per-protocol analysis (Table 2).

Table 2: Success rates in different groups according to Intention-to-treat and Per-protocol principles

\begin{tabular}{|c|c|c|c|c|c|c|c|c|}
\hline \multirow[b]{2}{*}{ Groups } & \multicolumn{4}{|c|}{ Intention-to-treat analysis } & \multicolumn{4}{|c|}{ Per-protocol analysis } \\
\hline & Success & Failure & Total & p-value & Success & Failure & Total & p-value \\
\hline Group 1 & $36(67.9 \%)$ & $17(32.1 \%)$ & $53(100.0 \%)$ & $<0.001$ & $36(67.9 \%)$ & $17(32.1 \%)$ & $53(100.0 \%)$ & $<0.001$ \\
\hline Group 2 & $44(93.6 \%)$ & $3(6.4 \%)$ & $47(100.0 \%)$ & $<0.001$ & $61(95.3 \%)$ & $3(4.6 \%)$ & $64(100.0 \%)$ & $<0.001$ \\
\hline Group 3 & $52(100.0 \%)$ & $0(0.0 \%)$ & $52(100.0 \%)$ & $\mathrm{n} / \mathrm{a}$ & $58(100.0 \%)$ & $0(0.0 \%)$ & $58(100.0 \%)$ & $\mathrm{n} / \mathrm{a}$ \\
\hline
\end{tabular}

$\mathrm{n} / \mathrm{a}-$ not applicable 
As insertion of UAS is not free of injury, 9 patients (5.9\%) had encountered ureteral wall injury. There was a grade 1 injury in 4 cases from group 1 . Among group 2 patients, 2 had grade 1 injury and 3 had grade 2 injury. None of the patients from group 3 had any injury.

\section{DISCUSSION}

Pre-stenting has been universally accepted as an easy and effective method of passive dilation facilitating UAS insertion during RIRS. However, not all the patient requires pre-stenting to negotiate the appropriate size of UAS. In a study of 248 patients by Mojilevkin Y et al. in 144 (58\%) patients, a 14F UAS could be passed easily. ${ }^{13}$ They have found that patients with an indwelling Double-J stent are 21 times more likely to accommodate a $14 \mathrm{~F}$ UAS. Besides, age was shown to be an independent predictor; older patients were more likely to accommodate the 14F UAS than younger patients. Similarly, in our study too younger patients had more failure than the older ones ( 32 vs 38 years).

In a study by Hameed D.A. et al., they have included 306 patients, whom they have divided into non-distensible and distensible ureters based on the ability of ureters to accommodate $10 \mathrm{~F}$ dilators. ${ }^{14}$ About $66.7 \%$ of their patients had distensible ureters. The failed ureters were passively dilated using a ureteric stent for 5-15 days before performing the secondary procedure. Those pre-stented patients had fewer minor and major complications than patients who did not receive a stent before stone treatment. The definition of the non-distensible or difficult ureter is not uniform in the literature. In our study about $67.92 \%$ of cases had distensible ureter, avoiding the need for dilatation prior to flexible ureteroscopy. But in a study by Cetti et al. about $8 \%$ of the ureters were difficult, not accommodating 11/13 F UAS. ${ }^{15}$ The Clinical Research Office of the Endourological Society URS Global Study collected prospective data for 1 year on consecutive patients with ureteric or renal stones treated with URS at 114 centers around the world. ${ }^{16}$ In their data of 1622 patients, $63.6 \%$ of the patients have distensible ureter not requiring pre-stenting for renal calculus. In a retrospective study by Zhang $\mathrm{J}$ et al., one hundred and seventysix flexible ureterorenoscopic (f-URS) procedures for unilateral renal stones were grouped into 2 categories depending upon pre-stenting. ${ }^{17}$ Successful primary access to the renal pelvis was achieved in 104 of $114(91.2 \%)$ patients without preoperative stenting, while all procedures with preoperative stenting $(n=62)$ were successfully performed.

Regarding the optimal duration of pre-stenting, none of the guidelines have any consensus. On the background of a large number of ureters $(67.92 \%)$ not requiring routine pre-stenting in our study, one and 2 weeks of pre-stenting is sufficient for $93.66 \%$ and $100 \%$ respectively. But the question here is, does it make any difference? Although the incidence of stent-related symptoms is beyond the scope of this study, the other conditions adversely affecting continuing pre-stenting for 2 weeks do not seem to be the major issue. At least $8 \%$ of the probability of nonaccommodating UAS in the ureter will be there if the patient would have undergone RIRS just after 1 week. In a recent retrospective study by Lee $\mathrm{MH}$ et al, they have categorized 560 patients into 3 groups similar to our study: Group 1- no pre-stenting, Group 2 - short pre-stenting ( $<7$ days), and Group 3 - long pre-stenting ( $>7$ days). The mean duration of pre-stenting in groups 2 and 3 was $4.9 \pm 0.9$ days and $9.7 \pm 3.2$ days respectively. About $85 \%$ of their patients had distensible ureters, while the rest of the patients in each group had undergone intraoperative active dilatation in $14.9 \%, 5.7 \%$, and $6 \%$ in groups 1,2 , and 3 respectively. ${ }^{18}$ In contrast to our study, the UAS in their study seems larger $12 / 14$ $\mathrm{F}$ and 14/16 F. But the rationale for using 14/16 F UAS with URF-P3 was not very clear. In the study by Zhang J et al., the duration of pre-stenting was not uniform. ${ }^{17}$ Some patients had pre-stenting for 7-40 days, while in case of failure, the second fURS were rescheduled for 4-34 days. Similarly, Hameed DA et al. had a pre-stenting duration of 5-21 days depending on when the patient could be returned to the operative schedule. ${ }^{14}$ Mahajan PM et al had a very small subset of patients (5.4\%) undergoing pre-stenting for RIRS with UAS. The duration of pre-stenting in his study was 4-15 days. ${ }^{19}$

During our study we were very gentle during the insertion of UAS. Failure of negotiation of UAS on the gentle force had led to tenting of that ureter. At the end of RIRS, the flexible ureteroscope was withdrawn along with the UAS with a complete circumferential assessment of the ureteral wall under vision. Our rate of ureteral wall injury was $5.9 \%$ with the absence of $3^{\text {rd }}$ and $4^{\text {th }}$ grade injuries. The incidence of ureteral wall injury in the original study by Traxer O. was $46.5 \%$ with the use of $12 / 14$ F UAS. ${ }^{4}$ Low grade injuries were found in 311 patients $(86.6 \%)$. There were grade 2, 3, and 4-grade injuries in $10.1 \%, 3.3 \%$, and $0.0 \%$ of patients, respectively. He stated that the pre-stenting vs no prestenting decreases the risk of severe injury by sevenfold and prestenting was associated with a significantly lower incidence of high-grade UAS related ureteral injuries. In the study by Hameed DA et al., among 306 patients, ureteric injury was found in $6.7 \%$ only. ${ }^{14}$ Ureteric injury was significantly associated with nondistensible ureters. They were managed with prolonged DJ stent placement for 1 month and on follow up none had evidence of ureteral stricture. In the retrospective study of Zhang J et al., with the use of $12 / 14 \mathrm{~F}$ UAS, their incidence of ureteral injury was 2.8 percent. ${ }^{17}$ Mild injuries were ignored in records of their operations, it was hard to know the accurate incidence of ureter injuries for all patients. But they noticed perforation of ureter or pelvis in $1.8 \%$ and $4 \%$ among stented and non-stented groups respectively. They further share their experience that if unreasonable resistance was perceived when passing through the ureter, it was better to terminate the fURS procedure rather than try with a greater force to avoid severe ureteric injury. In a retrospective study of 550 ureterorenoscopy by Lumma PP et al., they noticed that prestented patients had fewer complications than patients who did not receive a stent before stone treatment (minor complications $4.7 \%$ vs. $9.4 \%$, major complications $0.6 \%$ vs. $1.6 \%$ ). ${ }^{20}$ Especially, ureter perforations occurred more frequently in the non-stented group $(2.7 \%$ vs. $9.4 \%)$. Minor complications were treated with stent placement at the end of the procedure. Major complications required immediate and extensive intervention in contrast to minor complications.

Although the rate of ureteric complications did not seem significantly high among the pre-stented and non-stented groups in a study by Lee MH et al., the frequency was high in the nonstented group. ${ }^{18}$ The UAS they used, was $12 / 14 \mathrm{~F}$. The overall complication rate was $5 \%$ with the distribution of $7 \%, 5.1 \%$, and $2.4 \%$ in non-stented, short-term stented and long-term stented groups respectively. Even the injuries of all grades up to 4 were higher in the non-stented group. Despite those results, the author denies that preoperative double-J stenting is necessary to prevent ureteral injuries during ureteroscopic surgery. In contrast, Traxer 
and Thomas found that the most significant predictor of severe ureteral injury was no placement of a Double-J stent before RIRS. Pre-stenting versus no pre-stenting decreased the risk of severe injury by sevenfold $(\mathrm{p}<0.001) .{ }^{4}$ Even the Clinical Research Office of the Endourological Society URS Global Study had concluded that for the renal stones, using a preoperative JJ stent, there was a trend for decreased intraoperative complications. ${ }^{16}$

We do have limitations in our study. The sample size in our study seems limited compared to the available literature. The force required to negotiate UAS is very subjective and difficult to describe. So, during RIRS, what we would like to suggest is that routine pre-stenting can be avoided. Adequate counseling should be done about the possibility of failure to accommodate UAS during RIRS. If so, pre-stenting for 2 weeks is the ultimate choice.

\section{CONCLUSIONS}

More than two-thirds of the ureters do not require presenting and are distensible enough to proceed for RIRS with the use of 10/12 F UAS. Whereas UAS placement was successful in $93.66 \%$ after one week and $100 \%$ after two weeks of presenting.

\section{REFERENCES}

1. Hussain M, Acher P, Penev B, Cynk M. Redefining the limits of flexible ureterorenoscopy. Journal of endourology 2011; 25:45-9. $\underline{\text { Website }}$

2. Alenezi H, Denstedt JD. Flexible ureteroscopy: Technological advancements, current indications and outcomes in the treatment of urolithiasis. Asian J Urol 2015;2:133-41. Website

3. Kourambas J, Byrne RR, Preminger GM. Does a ureteral access sheath facilitate ureteroscopy? The Journal of urology 2001; 165:78993. Website

4. Traxer O, Thomas A. Prospective evaluation and classification of ureteral wall injuries resulting from insertion of a ureteral access sheath during retrograde intrarenal surgery. The Journal of urology 2013; 189:580-4. Website

5. De Coninck V, Keller EX, Rodríguez-Monsalve M, Audouin M, Doizi S, Traxer O. Systematic review of ureteral access sheaths: facts and myths. BJU Int 2018; 122:959-69. Website

6. Roberts WW, Cadeddu JA, Micali S, Kavoussi LR, Moore RG. Ureteral stricture formation after removal of impacted calculi. The Journal of urology 1998; 159:723-6. Website

7. Hubert KC, Palmer JS. Passive dilation by ureteral stenting before ureteroscopy: eliminating the need for active dilation. The Journal of urology 2005; 174:1079-80. Website

8. Astroza G, Catalán M, Consigliere L, Selman T, Salvadó J, Rubilar F. Is a ureteral stent required after use of ureteral access sheath in presented patients who undergo flexible ureteroscopy? Cent European J Urol 2017; 70:88-92. Website

9. Chu L, Farris CA, Corcoran AT, Averch TD. Preoperative stent placement decreases cost of ureteroscopy. Urology 2011;78:309-13. Website

10. Pollard SG, Macfarlane R. Symptoms arising from Double-J ureteral stents. The Journal of urology 1988; 139:37-8. Website

11. Joshi HB, Stainthorpe A, MacDonagh RP, Keeley FX, Jr., Timoney AG, Barry MJ. Indwelling ureteral stents: evaluation of symptoms, quality of life and utility. The Journal of urology 2003; 169:1065-9; discussion 9. Website

12. Schulz KF, Altman DG, Moher D. CONSORT 2010 statement: updated guidelines for reporting parallel group randomised trials. BMJ (Clinical research ed) 2010; 340:c332. Website

13. Mogilevkin Y, Sofer M, Margel D, Greenstein A, Lifshitz D. Predicting an effective ureteral access sheath insertion: a bicenter prospective study. Journal of endourology 28:1414-7. Available from: Website

14. Hameed DA SA, Osman MM, Gadelmoula MM, Kurkar A, Elgammal MA (2017) Outcome of ureteral distensibility on the success of ureteroscopy: A prospective hospital-based descriptive study. African Journal of Urology 2014; 23:33-7. Website

15. Cetti RJ, Biers S, Keoghane SR. The difficult ureter: what is the incidence of pre-stenting? Ann R Coll Surg Engl 2011; 93:31-3. Website

16. Assimos D, Crisci A, Culkin D, Xue W, Roelofs A, Duvdevani M, et al. Preoperative JJ stent placement in ureteric and renal stone treatment: results from the Clinical Research Office of Endourological Society (CROES) ureteroscopy (URS) Global Study. BJU Int 2016; 117:64854. Website

17. Zhang J, Xu C, He D, Lu Y, Hu H, Qin B, et al. Flexible ureteroscopy for renal stone without preoperative ureteral stenting shows good prognosis. PeerJ 2016; 4:e2728. Website

18. Lee MH, Lee IJ, Kim TJ, Lee SC, Jeong CW, Hong SK, et al. The effect of short-term preoperative ureteral stenting on the outcomes of retrograde intrarenal surgery for renal stones. World J Urol 2019; 37:1435-40. Website

19. Mahajan PM, Padhye AS, Bhave AA, Sovani YB, Kshirsagar YB, Bapat SS. Is stenting required before retrograde intrarenal surgery with access sheath. Indian J Urol 2009; 25:326-8. Website

20. Lumma PP, Schneider P, Strauss A, Plothe KD, Thelen P, Ringert RH, et al. Impact of ureteral stenting prior to ureterorenoscopy on stonefree rates and complications. World J Urol 2013; 31:855-9. Website 